\title{
The Definitions of Information Science in China ${ }^{\dagger}$
}

\author{
Xiaoying Zhou *, Xiaoning Yu and Lu Zhang \\ School of Information Resources Management, Renmin University of China, Beijing 100872, China; \\ iyuxiaoning@163.com (X.Y.); zhanglu0395@163.com (L.Z.) \\ * Correspondence: zhouxiaoying@ruc.edu.cn \\ † Fourth International Conference on Philosophy of Information, Berkeley, California, USA, 2-6 June 2019. \\ Published: 30 June 2020
}

\begin{abstract}
This paper collected 128 information science definitions circulating in China from three different sources. By counting the word frequency and generating a word cloud map of the 128 definitions, the high frequency words in the definitions of information science and scholars' understanding of information science are analyzed. Through an analysis of the characteristics of the 128 information science definitions, this paper summarizes the four basic patterns and three compound patterns of information science definitions, and analyzes the connotations and development characteristics of these definitions of information science in China.
\end{abstract}

Keywords: information science definition; characteristics analysis; basic patterns analysis

\section{Introduction}

This paper collected 128 information science definitions circulating in China from three different sources. By counting the word frequency and generating a word cloud map of the 128 definitions, the high frequency words in the definitions of information science and scholars' understanding of information science are analyzed. Through an analysis of the characteristics of the 128 information science definitions, this paper summarizes the four basic patterns and three compound patterns of information science definitions, and analyzes the connotations and development characteristics of these definitions of information science in China.

\section{Data Sources}

This study collected information science definitions circulating in China from three sources, the China Academic Journals Full-text Database of CNKI (China National Knowledge Infrastructure) [1], Chaoxing e-Book Database [2] and the reference book (Cihai, etymology [3], encyclopedia, dictionary [4]). After data de-reprocessing, 128 of them were retained for analysis and research.

\section{Analysis}

\subsection{Time Series Analysis}

We conducted a time series statistical analysis of the definitions of information science from three perspectives.

The publication times of 128 information science definitions were counted and the number of information science definitions in each year was sorted, then a publication sequence diagram of the definitions of information science was made, organized by the specific year and order of publication.

According to the definitions of information science, the timing chart was divided into four stages. The word frequency statistics of the information science definitions in each stage and the top 10 keywords were listed. To produce a phased word frequency statistics table in order to identify hot 
topics within the definitions of information science at different stages and the overall trend of these definitions, the time was divided into five periods, and the top 30 high-frequency word timing change line graphs are defined according to the word frequency time series distribution of the five periods.

\subsection{Dimensional Analysis}

Among the 128 definitions, 116 of them contained the phrase "Information science is...", and after classification and statistics, they can be divided into eight groups as shown in the table. It can be seen that for the dimension of "What is information science", the definition description is mostly related to "science" and "discipline". In total, 108 of them contained research objects, and highfrequency words are intelligence, knowledge, and information. Among the 108 definitions, 76 definitions contained specific submission times. Statistics were obtained on the timing and frequency of the three keywords of intelligence, information and knowledge in these definitions. The results are shown below. It can be seen that, overall, the term "intelligence" has an absolute advantage in all periods, and the frequency of information and knowledge increased slightly in the middle and late stages. Overall, 94 of them contain research content. After the de-stop words and word segmentation were processed, 384 keywords were obtained. Further analysis shows that the research content of information science is mainly divided into two categories. Finally, 46 of them contained the attributes of information science. After the de-stop words and word segmentation were processed for the attribute descriptions in these definitions, 54 keywords were obtained.

After further summarizing, the definitions were divided into five categories: scientific, comprehensive, characteristic, new, and other. On the whole, the descriptions of the attributes of information science are mainly reflected in the scientific, comprehensive, characteristic performance and emerging nature of the subject.

Among the 128 definitions, 64 of them contained research objectives. After the de-stop words and word segmentation were processed for the attribute descriptions in these definitions, the results are shown below.

According to the above quantitative word frequency statistics results, combined with the research objectives in the 65 definitions, qualitative analysis reveals that the research objectives of information science are mainly concentrated in the following aspects:

The first, to analyze and explain the principles, theories, methods, and laws in the process of information life cycle management;

The second, to maximize the use of information by users;

The third, to provide support and guidance for problem solving and decision making.

\section{Conclusions: Modes of Information Science Definition}

The definition modes are divided into three aspects: subject attribute, research object/content and research purpose/task. Only a small number of the definitions are based on the above three aspects. Most definitions integrate some aspects. The description is difficult to distinguish, so the addition of several combination modes must be considered. Finally, the following eight modes are summarized:

- Embodying the Subject Attribute;

- Reflecting the Research Object/Content;

- Clearing the Research Purpose/Task;

- Embodying Subject Attribute and Reflecting the Research Object/Content;

- Embodying the Subject Attribute and Clearing the Research Purpose/Task;

- Embodying the Subject Attribute, Reflecting the Research Object/Content and Clearing the Research Purpose/Task;

- Reflecting the Research Object/Content and Clearing the Research Purpose/Task;

- Description of Features. 
Author Contributions: Methodology, X.Z.; formal analysis, X.Y.; investigation, L.Z.; data curation, X.Y.; writing-original draft preparation, X.Y.; writing-review and editing, L.Z.; supervision, X.Z.; project administration, X.Z. All authors have read and agreed to the published version of the manuscript.

Funding: This research was funded by CNSF of China, grant number 71473260.

\section{References}

1. CNKI China Academic Journals Full-Text Database. Available online: https://www.cnki.net (accessed on 3 April 2019).

2. Chaoxing e-Book Database. Available online: http://www.sslibrary.com (accessed on 3 April 2019).

3. Editorial Committee of Cihai. Cihai Miniature, 1989th ed.; Shanghai Lexicographic Publishing House: Shanghai, China, 1990; p. 981.

4. Editorial Board of Encyclopedia of China. Encyclopedia of China, 2nd ed.; Encyclopedia of China Publishing House: Beijing, China, 2009; p. 188.

(C) 2020 by the authors. Licensee MDPI, Basel, Switzerland. This article is an open access article distributed under the terms and conditions of the Creative Commons Attribution (CC BY) license (http://creativecommons.org/licenses/by/4.0/). 